\title{
Языковая политика Франции: некоторые правовые аспекты
}

Жан-Франсуа Бальди

DOI: 10.30547/mediaalmanah.6.2020.144151

(c) Бальди Жан-Франсуа заместитель руководителя

Национального управления по продвижению французского языка и языков Франции, Министерство культуры Франции (г. Париж, Франция)
Юридические рамки, в которые вписывается языковая политика во Франции, относятся в первую очередь к политике в отношении французского языка, но в то же время касаются политики, поддерживающей языковое разнообразие, поскольку вопросы, связанные с французским языком, нельзя отделить от проблем, имеющих отношение к другим языкам, на которых говорят во Франции.

Становление и развитие французской нации шло в тесной связи с развитием национального языка, поэтому политика в отношении французского языка имеет долгую историю, и вехи этой истории известны. Ее начало - 1539 г., когда король Франции Франциск I подписал Ордонанс Виллер-Котре, согласно которому французский язык стал официальным языком права и администрации, заняв место латыни и других языков в стране.

Прошло более четырех столетий, и в 1992 г. в Конституцию страны было введено положение, которое гласило, что «языком Республики является французский язык». Остановимся подробнее на статье $2 \mathrm{Koн-}$ ституции Франции, потому что она - основа нашей политики и имеет весьма конкретные последствия.

Статья 2 помещена в Раздел 1 Конституции - «О суверенитете». Таким образом, французский язык является атрибутом 


\section{La politique linguistique en France: le cadre juridique}

Jean-François Baldi

(C) Baldi Jean-François

Délégué général adjoint à la langue française et aux langues de France,

Ministère de la Culture de France

(Paris, France)

La politique du français est placée dans le temps long de l'histoire et, en France, la Nation s'est construite dans un rapport étroit avec la langue française.

Les jalons de cette histoire sont connus, à commencer par l'année 1539, où le roi de France François ler signe l'ordonnance de Villers-Cotterêts, qui fait du français la langue officielle du droit et de l'administration, en lieu et place du latin et des autres langues du pays.

Si l'on fait un saut de plus de quatre siècles, nous arrivons à l'année 1992, avec l'inscription dans la Constitution française-donc dans le texte le plus élevé dans la hiérarchie des normes-de la disposition qui prévoit que "La langue de la République est le français ».

Il s'agit de l'article 2 de notre Constitution, sur lequel il convient de se pencher attentivement, parce qu'il est le socle de notre politique et qu'il a des implications très concrètes.

D'abord, cet article 2 prend place dans le titre premier de la Constitution, intitulé « De la souveraineté ».

La langue française est donc un attribut de la souveraineté de notre pays, au même titre que son emblème national (le drapeau bleu, blanc, rouge), son hymne (La Marseillaise), sa devise ("Liberté, égalité, fraternité ») et son principe ("Gouvernement du peuple, par le peuple et pour le peuple »).

Par ailleurs, cet article a des implications concrètes pour l'Etat, qui résultent de la jurisprudence du Conseil constitutionnel suite à des contentieux sur lesquels il a eu à se pencher.

Quatre conséquences.

Les deux premières illustrent une conception unitaire, étatique, centralisée de la langue française.

Première conséquence, l'usage du français s'impose aux personnes morales de droit public (autrement dit les personnes morales telles que l'Etat et les collectivités territoriales, qui sont soumises au droit public) et aux personnes de droit privé dans leur mission de service public

Seconde conséquence, les particuliers ne bénéficient pas, dans leurs relations avec l'administration et les services publics, d'un droit à l'usage d'une langue autre que le français, et ne peuvent être contraints à un tel usage. 
суверенитета нашей страны, так же, как и национальная эмблема (флаг: синий, белый, красный), гимн («Марсельеза»), девиз («Свобода, Равенство, Братство») и принцип («Правление народа, народом и для народа»). Кроме того, эта статья имеет конкретное влияние на государство, которое вытекает из судебной практики Конституционного совета как следствие рассмотренных им споров. Два первых из них иллюстрируют унитарную, государственную, централизованную концепцию французского языка.

Первое следствие - использование французского языка обязательно для юридических лиц публичного права (иными словами, юридических лиц, таких как государство и территориальная единица, к которым применимо публичное право), а также и для лиц частного права на государственной службе.

Второе - частные лица в отношениях с администрацией и государственными службами не имеют права использовать какой-либо язык, кроме французского, и не могут принуждаться к его использованию.

Поясним на примере: гражданин, который владеет бретонским, обращается в префектуру в Бретани и может обратиться к сотруднику префектуры на бретонском, но эта практика никоим образом не гарантируется правом. Сотрудник префектуры, со своей стороны, может по собственной инициативе ответить ему на бретонском, но он с точки зрения права тоже не обязан этого делать.

Два других, напротив, придают более либеральный оттенок языковому праву в нашей стране.

Во-первых, использование переводов никоим образом не запрещено: так, государственные службы могут использовать другой язык, кроме французского, при условии, что существует версия на французском языке и только она будет иметь юридическую силу в случае спора.

Во-вторых, статья 2 Конституции не должна приводить к неверному пониманию важности свободы слова и выражения мнений, свободы предпринимательской деятельности и свободы торговли и промышленности.

Основываясь на высшем принципе свободы слова и выражения мнений, Конституционный совет посчитал не соответствующим Конституции любое категорическое требование для телеканалов или радиостанций по использованию официальных терминов или выражений, одобренных постановлениями министерства.

Из этой 2 статьи Конституции, крометого, вытекает совокупность правовых норм, которые в основном собраны в ссылках на правовые акты. Речь о законе от 4 августа 1994 г. об использовании французского языка, который также называют законом Тубона (по имени министра культуры, который подготовил проект закона и представил его в парламенте).

Если бы нужно было резюмировать общую цель этого закона, можно было бы сказать, что он позволяет реализацию «права на французский язык» на территории Франции для наших граждан и всех тех, кто принял решение изучать французский язык. Это право говорить и получать информацию на французском языке реализуется в нескольких сферах: потребление, занятость, образование, реклама, транспорт, научные симпозиумы. Введение обязательного использования французского языка во многих ситуациях повседневной жизни позволяет, в частности, обеспечивать здоровье и безопасность потребителей, сотрудников и граждан, получать знания ученикам, студентам и ученым.

Нужно уточнить одно: поскольку речь идет о межведомственном механизме, применение этого закона входит в полномочия не нашей службы, а администрации. Например, в том, что касается информирования потребителей, задача контролировать применение закона возлагается на уполномоченные службы Министерства экономики. 
Un exemple permettra de mieux comprendre. Un citoyen, locuteur de breton, qui se trouve à un guichet d'une préfecture en Bretagne, peut choisir de s'adresser en breton à l'employé de la préfecture, mais il ne s'agit en aucun d'une pratique garantie par le droit. L'employé de la préfecture peut quant à lui, de sa propre initiative, lui répondre en breton, mais il n'y est pas non plus tenu par le droit.

Deux autres éléments de la jurisprudence du Conseil constitutionnel donnent, au contraire, une couleur plus libérale à notre droit linguistique.

D'abord, l'utilisation de traductions n'est en rien prohibée: il est ainsi possible, pour les services publics, de recourir à une autre langue que le français, à la condition qu'une version française existe, qui seule fait foi en cas de litige

Ensuite, l'article 2 de notre Constitution ne doit pas conduire à méconnaître l'importance que revêt la liberté d'expression et de communication, la liberté d'entreprendre et la liberté du commerce et de l'industrie.

C'est en se fondant sur le principe supérieur de liberté d'expression et de communication que le Conseil constitutionnel a estimé contraire à la Constitution toute obligation faite aux chaînes de télévision ou de radio de recourir à des termes ou des expressions officiels approuvés par des arrêtés ministériels.

De cet article 2 de la Constitution découle par ailleurs un ensemble de dispositions légales, qui sont pour l'essentiel réunies dans un texte de référence. Il s'agit de la loi du 4 août 1994 relative à l'emploi de la langue française, appelée loi Toubon du nom du ministre de la culture qui a préparé ce texte et l'a défendu devant le Parlement. Nos célébrons d'ailleurs cette année (pour quelques jours encore), les 25 ans de l'adoption de ce texte.

S'il fallait résumer d'une formule la visée d'ensemble de cette loi, on peut dire qu'elle permet l'exercice d'un « droit au français » sur notre territoire, au profit de nos concitoyens et de tous ceux qui ont fait le choix d'apprendre notre langue.

Ce droit à s'exprimer et à recevoir une information en français s'exerce dans plusieurs domaines :la consommation, le travail, l'enseignement, la publicité, les transports, les colloques scientifiques. Imposer l'emploi de la langue française dans un grand nombre de situations de la vie quotidienne permet notamment d'assurer la santé et la sécurité des consommateurs, des salariés et des citoyens, de favoriser l'accès aux savoirs des élèves, des étudiants et des chercheurs.

Une chose doit être précisée: étant un service à vocation interministériellej'y reviendrai à la fin de mon propos - l'application de ce texte ne relève pas de notre service, mais des administrations concernées par tel ou tel domaine visé par la loi. Par exemple, en ce qui concerne l'information du consommateur, ce sont les services compétents du ministère de l'économie qui sont chargés de contrôler l'application de la loi.

J'ajoute que notre droit impose aux services publics des contraintes particulières en matière d'emploi de la langue française. Ainsi, les administrations de l'Etat sont tenues d'employer les termes et expressions publiés au Journal officiel dans le cadre de ce qu'on appelle le dispositif interministériel d'enrichissement de la langue française.

Indépendamment de cette terminologie officielle, il est attendu des services de l'Etat une exemplarité dans l'emploi de la langue française dans les outils d'information et de communication, ainsi que dans les situations de communication internationale. 
Добавлю, что наше законодательство налагает на государственные службы определенные ограничения в том, что касается использования французского языка. Так, государственная администрация обязана использовать термины и выражения, опубликованные в Официальном бюллетене Французской Республики. В рамках того, что называют «межведомственным механизмом обогащения французского языка», создаются новые слова и выражения для заполнения пропусков в словаре нашего языка и обозначения по-французски концепций и явлений, которые возникают в иностранных языках. Использование этой «официальной терминологии» не является обязательным для граждан, согласно принципу свободы слова, но оно является обязательным для государственной администрации.

Независимо от этой официальной терминологии от государственных служб ожидается образцовое использование французского языка в средствах информации и коммуникации, а также в ситуациях международного общения. Хотя она и является весьма цельной, но эта правовая норма иногда вызывает недопонимание у некоторых наших сограждан или же за рубежом, где ее могут интерпретировать как запрет на использование во Франции других языков, кроме французского.

Соответственно, мы постоянно разрушаем этот миф: закон 1994 г. не запрещает использование региональных языков, что ясно обозначено, и также не запрещает говорить на иностранных языках. Закон также не имеет целью охранять чистоту французского языка, ограничивая использование иностранных слов: он касается сфер употребления французского языка, а не его содержания.

Теперь рассмотрим правовую динамику. В 2013 г. после крайне оживленной дискуссии в стране закон позволил при соблюдении определенных условий вести преподавание в высших учебных заведениях на иностранных языках. Речь идет об образовательных программах, составленных в рамках соглашений с иностранными университетами, или о программах, получающих финансирование от Европейского союза. Тогда правительство аргументировало это тем, что необходимо ввести в образовательную систему гибкость, которая позволит привлечь больше студентов, в частности из Индии, поскольку перспектива получать образование на английском языке может повлиять на их решение продолжать учебу во Франции.

Эти изменения в том, что касается продолжения использования французского языка, сочетаются с другими положениями, из-за которых распространенность иностранных языков в нашей стране снижалась. Имеется в виду реформа тестирования по языкам на вступительных экзаменах в Национальную школу администрации, где обучается будущая административная элита нашей страны. Сейчас в рамках экзамена можно сдать только английский язык, хотя раньше можно было выбрать один из восьми языков (и среди них русский язык).

Эта мера на первый взгляд может показаться незначительной. В действительности в ней можно усмотреть конкретное «послание». Оно заключается не в том, что восхождение по социальной лестнице невозможно без английского языка, ведь в конце концов в этом есть доля истины, а в том, что оно возможно лишь благодаря владению только английским языком, исключая все остальные языки.

В целом эти постоянные меры могли способствовать тому, что у общественности сложилось своего рода неприятие французского языка, который не считается современным языком. Атакже тому, что английский язык видится в благоприятном свете, подспудно играет роль языка, без которого невозможно быть частью современного мира.

Тем не менее, если считать, что стакан наполовину полон, а не наполовину пуст, 
S'il présente une forte cohérence, ce cadre juridique suscite parfois des incompréhensions chez un certain nombre de nos concitoyens ou encore à l'étranger, où il peut être interprété comme interdisant l'usage en France d'autres langues que le français.

Nous ne cessons donc de dissiper ce malentendu: la loi de 1994 ne s'oppose pas à l'emploi des langues régionales, elle le stipule d'ailleurs explicitement, et elle ne s'oppose pas davantage à l'emploi des langues étrangères.

Elle n'a pas non plus vocation à préserver la pureté du français en faisant la chasse aux mots étrangers : elle porte sur la présence du français et non sur son contenu.

Plaçons-nous maintenant au plan des dynamiques juridiques: qu'observe-t- on?

Il ne faut pas masquer la réalité: on constate depuis 25 ans un retrécissement régulier du champ d'application de la loi de 1994. Plusieurs exceptions à l'obligation d'emploi du français ont été adoptées.

Je ne vais pas les égréner, car elles sont de portée très variables. Je me contenterai de citer en 2012 les documents de construction, de maintenance et de pilotage des avions.

Plus grave, en 2013, à l'issue d'un très vif débat dans notre pays, une loi a autorisé, sous certaines conditions, des enseignements en langues étrangères dans l'enseignement supérieur. Il s'agit d'enseignements dispensés dans le cadre d'accords avec des universités étrangères ou de programmes financés par l'Union européenne.

L'argument du gouvernement de l'époque était d'introduire dans le système éducatif une souplesse indispensable pour attirer davantage d'étudiants, notamment asiatiques ou indiens, que la perspective de formations en anglais pouvait conforter dans leurs décisions de poursuivre leurs études en France.

Ces brèches ouvertes dans la préservation de l'emploi du français font système avec d'autres dispositions qui ont tendu à réduire la présence des langues étrangères dans notre pays. Je pense à la réforme de l'épreuve de langues au concours d'entrée à l'École nationale d'administration-une école qui forme les élites administratives de notre pays. C'est désormais la seule langue anglaise qui est proposée à cette épreuve, alors que jusqu'alors huit langues étrangères étaient proposées, dont le russe.

Cette mesure peut paraître mineure. En réalité, elle renvoie le message, non pas que l'ascension sociale passe par la maîtrise de l'anglais, car après tout ce message n'est pas entièrement faux, mais que cette ascension ne passe que par la seule maîtrise de l'anglais, à l'exclusion de toute autre langue.

Plus généralement, ces reculs successifs ont pu contribuer à créer dans l'opinion une forme de défiance envers le français-supposé ne pas être une langue de la modernité-et renforcer les représentations favorables à l'anglais, implicitement désigné comme la langue sans laquelle il n'est pas possible d'entrer dans cette modernité.

Toutefois, si l'on regarde le verre à moitié plein et non le verre à moitié vide, retenons que le dispositif législatif en faveur de la langue française a été dans l'ensemble préservé, même s'il a été écorné sur quelques points sensibles.

Je l'ai dit en préambule, notre politique ne se limite pas à la seule langue française. Deux autres dispositions constitutionnelles orientent cette politique. 
можно отметить, что правовые рамки в отношении французского языка в целом были сохранены, хотя и утратили несколько значительных пунктов. Наша политика не ограничивается только французским языком. Направление этой политике задают еще два положения Конституции.

Статья 75-1 Конституции гласит: «Региональные языки являются частью национального достояния Франции». Эта статья, введенная в Конституцию 23 июля 2008 г., имеет большое символическое значение, поскольку в ней признается, что наша страна отличается значительным языковым богатством. Однако конкретный охват этого закона небольшой. Речь идет о некоем заявлении, которое нельзя применить при вынесении судебного решения, в отличие от статьи Конституции о французском языке.

Также в 2008 г. в Конституцию вошло понятие «франкофонии». Речь о статье 87: «Республика участвует в развитии солидарности и сотрудничества между государствами и народами, общим для которых является французский язык». Эта статья касается главным образом политической сферы. Во-первых, она показывает степень ответственности Французской Республики в проявлении солидарности между франкоговорящими странами. Во-вторых, она придает конституционную ценность понятию «общим для которых является французский язык»; в этом отношении она ставит общность языка в основание деятельности нашей страны во франкоязычном пространстве.

Итак, наша языковая политика опирается на три основных положения, основанные на Конституции (а конструкция на трех точках опоры всегда самая устойчивая):
- французский язык как фактор социальной сплоченности, наше общее благо;

- региональные языки как важнейшая часть нашего нематериального наследия, которые мы должны поддерживать;

- франкофония как инструмент продвижения французского языка в мире.

Политика не ограничивается документами, для ее последовательного проведения нужна четкая организация деятельности. Такую организацию обеспечивает наша служба - Национальное управление по продвижению французского языка и языков Франции. В ее задачи входит направление и координация языковой политики государства.

В связи с этим хотелось бы настаивать на использовании слов «направление» и «координация»; они позволят понять, как мы работаем и что характеризует нашу службу:

- это не «громоздкая» служба, у нас работает около 30 сотрудников;

- это политическая служба, а не классическая административная;

- это служба при Министерстве культуры (что имеет большое символическое значение: это означает, что язык не инструмент коммуникации, а в первую очередь воплощение культуры);

- наконец, что крайне важно, это межведомственная служба, сила которой исходит из ее способности задействовать государственные механизмы, а также всех акторов гражданского общества, к которым относится политика французского языка и языкового разнообразия.

Такая способность задействовать механизмы неразрывно связана с профессиональной оценкой всех вопросов, касающихся языковой политики (сточки зрения права, науки, дипломатии). 
D'abord l'article 75-1 de la Constitution, qui dispose que « "Les langues régionales appartiennent au patrimoine de la France ».

Cet article a été introduit le 23 juillet 2008 dans notre Constitution. Il a une valeur symbolique forte, car il reconnaît que notre pays est riche de la diversité des langues qui le constituent. En revanche, sa portée concrète est faible. Il s'agit d'une disposition essentiellement déclarative, qui n'est pas opposable à un tiers dans une décision de justice, à la différence de l'article de la Constitution relatif à la langue française.

La Francophonie a également fait son entrée dans la Constitution en 2008. Il s'agit de l'article 87 : « La République française participe au développement de la solidarité et de la coopération entre les États et les peuples ayant le français en partage ».

Cet article a une portée essentiellement politique. D'abord, il illustre la responsabilité de la République française dans le développement des solidarités francophones. Ensuite, il donne une valeur constitutionnelle à la notion de " français en partage "; à ce titre, il met le lien fondateur de la langue au coeur de l'action de notre pays au sein de la Francophonie.

Notre politique linguistique s'appuie donc sur trois piliers inspirés de notre Constitution (et l'on est toujours stable sur trois piliers):

- la langue française, notre bien commun, facteur de cohésion sociale

- les langues régionales, dimension essentielle de notre patrimoine immatériel, que nous devons nous attacher à faire vivre

- la francophonie, à savoir la langue française dans la diversité de ses expressions dans le monde.

Une politique ne se limite pas à des textes, elle dépend aussi d'une organisation pour les mettre en œuvre. Cette organisation trouve en son cœur notre service, la Délégation générale à la langue française et aux langues de France, qui est chargée d'orienter et de coordonner la politique linguistique de l'État.

- c'est un service " léger ", d'une trentaine d'agents,

- c'est un service politique, et non un service administratif classique.

- c'est un service du ministère de la culture. Ce rattachement à un sens symbolique fort. Il signifie que la langue, avant d'être un outil de communication, est aussi et d'abord l'incarnation d'une culture.

- enfin, et surtout, c'est un service à vocation interministérielle, dont la force tient à sa capacité à mobiliser tous les rouages de l'Etat, et au-delà tous les acteurs de la société civile concernés par la cause du français et de la diversité linguistique.

Cette capacité de mobilisation est indissociable d'une capacité d'expertisejuridique, scientifique, diplomatique-sur tous les sujets ayant trait aux langues. Capacité qui m'a donné le plaisir de vous présenter le cadre légal dans lequel nous travaillons à la délégation générale à la langue française et aux langues de France.Je vous remercie de votre attention. 